Z. Yin, I. Boxx, M. Stöhr, O. Lammel, and W. Meier, Confinement-induced instabilities in a jet-stabilized gas turbine model combustor, Flow, Turbulence and Combustion 98 (2017) 217-235.

The original publication is available at www.springerlink.com

http://dx.doi.org/10.1007/s10494-016-9750-5 


\title{
Confinement-induced instabilities in a jet-stabilized gas turbine model combustor
}

\author{
Zhiyao Yin · Isaac Boxx • Michael Stöhr . \\ Oliver Lammel · Wolfgang Meier
}

Received: date / Accepted: date

\begin{abstract}
Self-sustained jet flapping is observed in a confined, premixed and preheated methane-air turbulent flame, generated in a single-nozzle jet-stabilized gas turbine model combustor designed based on the FLOX ${ }^{\circledR}$ concept ${ }^{1}$. The flapping frequency and its complex motion within the confinement of the combustor are characterized in detail using proper orthogonal decomposition (POD) of the flow fields measured by particle imaging velocimetry (PIV). The influence of jet flapping on combustion stability is examined using simultaneous $\mathrm{PIV} / \mathrm{OH}$ chemiluminescence imaging and PIV/planar laser-induced fluorescence of $\mathrm{OH}$ radicals (OH PLIF) at $5 \mathrm{kHz}$ repetition rate. By influencing the size and location of the recirculation zones, jet flapping modifies the flame shape and flame lift-off height. It also controls the amount of hot gas entrainment into the recirculation zones. In extreme cases, jet flapping is found to cause temporary local extinction of the flame, due to jet impingement on the combustor wall and partial blockage of burned gas entrainment. The flame is only able to recover after the jet detaches from the wall and reopens the back flow channel. The results suggest that jet flapping could play a key role in the stabilization mechanisms in similar jet-stabilized combustors.
\end{abstract}

Keywords Confined Turbulent Flames · Jet Stabilized Flames · Jet Oscillation · Highspeed Diagnostics · Proper Orthogonal Decomposition

\section{Introduction}

In recent years, a series of jet-stabilized combustors based on the FLOX ${ }^{\circledR}$ concept have been developed at DLR and have been the subjects of extensive experimental $[1-8]$ and numerical studies $[9,10]$. The concept is regarded as a viable alternative to

Zhiyao Yin $(\bowtie) \cdot$ Isaac Boxx $\cdot$ Michael Stöhr $\cdot$ Oliver Lammel $\cdot$ Wolfgang Meier

Institute of Combustion Technology, German Aerospace Center (DLR), 70569, Stuttgart, Germany E-mail: zhiyao.yin@dlr.de

1 FLOX $^{\circledR}$ is a registered trademark of WS Wärmeprozesstechnik GmbH, Renningen, Germany. 
swirl-stabilized flames due to its high resistance to thermoacoustics and flame flashback [1], while also attaining high fuel flexibility and low NOx production [2]. Without alluding to the flameless [11], volume or mild combustion [12], FLOX ${ }^{\circledR}$ combustors refer exclusively to combustors designed following a core principle: flames are stabilized by intense mixing of the reactants and products from strong flow recirculations created by high momentum jet(s) discharged into the combustor chamber. A series of combustors with various nozzle configurations have so far been tested (at either room or elevated pressures): (1) combustors operate with (partially) premixed air and gaseous fuels (mainly methane and hydrogen), and features either a single nozzle [3], linearly aligned multiple nozzles [5] or circularly arranged multiple nozzles with [4] and without a pilot flame [1, 2, 8]; (2) combustors operate with liquid fuels and incorporate either a single nozzle or multiple nozzles [6]. In particular, the single-nozzle FLOX ${ }^{\circledR}$ combustor developed in Ref.[3] has been aimed at isolating the interactions between a single jet and the recirculation zone, in pursuit of the fundamental mechanisms of flame stabilization of the FLOX ${ }^{\circledR}$ concept. The latest discovery of pronounced periodic jet oscillations in the same single-nozzle combustor [7] has drawn fresh attention to the potential sources of instabilities in FLOX ${ }^{\circledR}$ combustors that may limit their operating range. It has also led to the speculation that individual jets in a multi-nozzle configuration may not simply act independently, but rather interact with each other due to jet oscillation, which could explain the occasional merging of flames sustained by different jets [8].

Laminar and turbulent jets under confinement exhibiting self-excited and selfsustained oscillation are well documented in the literature [13-15]. The common understanding is that, this type of oscillation is triggered by obstructed shear layers, and is sustained by a feedback loop between initial disturbances and the impinging points. The occurrence and behavior of jet oscillation depends heavily on the jet velocity and confinement configuration [13]. Jet flapping and jet precession (defined as rotation of the entire jet with respect to the nozzle axis) have been identified as the primary patterns of oscillation in confined jets. The former usually occurs in planar jets under rectangular confinement [15-18]. The latter has been encountered in cases of round jets expanding into a concentric cylindrical chamber, which also induces a swirling flow within the confinement [19-21]. Jet oscillation can exert a profound influence on the flow field, particularly on the redistribution of turbulent kinetic energy. It can significantly increase jet spreading rate and enhance large scale entrainment of the ambient fluid while suppressing fine scale mixing [16]. In reacting flows, these features have been linked to a string of advantages [22]: increased flame volume compared with a non-oscillating jet; decrease in flame temperature and NOx production due to reduced global flame strain. Despite enduring interest in jet oscillation in various research fields, its implications on combustion stability have thus far received little scrutiny.

In order to understand the impact of jet oscillation on FLOX ${ }^{\circledR}$ combustors, measurements are carried out in this work to focus on: (1) the characterization of the jet oscillation pattern unique to the FLOX ${ }^{\circledR}$ design concept and (2) the analysis of its influence on combustion. The single-nozzle combustor where jet oscillation is first discovered [7] is used in this work for its ease of operation and its good optical accessibility. Although jet oscillation has since been observed in a wide range of air-fuel 
Fig. 1

Schematics of the single-nozzle combustor:

(a) Combustor chamber

(b-d) Front, side and top views of the bottom section of the combustor chamber

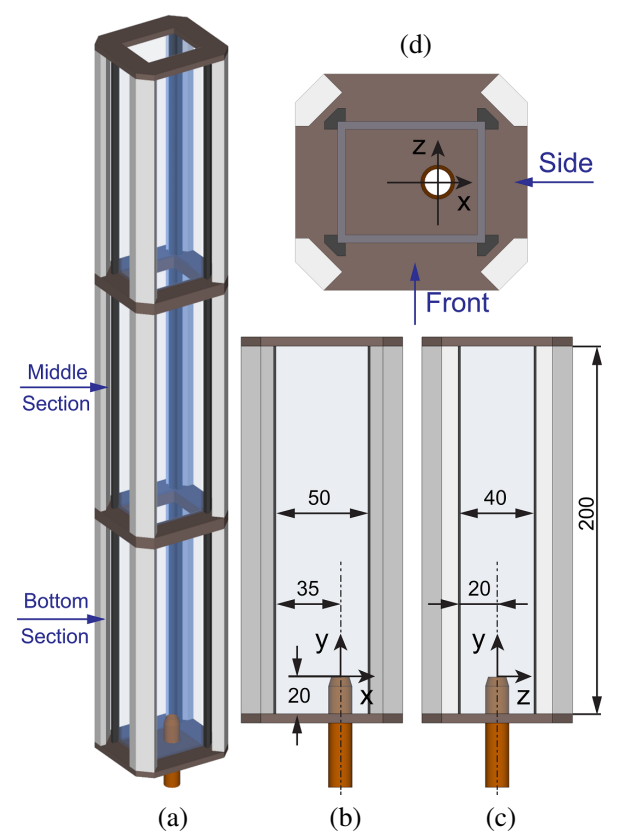

(a) (c)

ratios (with methane and/or hydrogen as fuel), this work concentrates on a standard test case (relevant to prior experimental and numerical studies $[3,7,9,10]$ ) with a preheated and fully premixed methane-air mixture at atmospheric pressure. Since jet oscillation scales with the Strouhal number (i.e., confinement geometry and jet velocity) $[13,15]$, the results from this work can be extended, at least qualitatively, to other operating conditions and other single-nozzle FLOX ${ }^{\circledR}$ combustors.

\section{Experimental}

\subsection{Combustor and operating condition}

Figure 1 shows schematically the single-nozzle FLOX ${ }^{\circledR}$ combustor used in this work. The combustor chamber consisted of three identical sections. Each was 200-mm tall and featured a rectangular cross section with an inner dimension of $50 \times 40 \mathrm{~mm}$. The jet nozzle was made of a straight stainless steel tube (ID=10 mm, L=400 mm). It had a chamfered tip and rose $20 \mathrm{~mm}$ above the base plate. It was offset from the geometric center of the combustor chamber, creating an asymmetry in the front view (Fig.1(b)). The off-center positioning of the jet was designed to imitate the situation around a single nozzle in a multi-nozzle FLOX ${ }^{\circledR}$ combustor, where nozzles were commonly placed near the chamber wall, leading to the formation of a large recirculation zone to one side of the jets $[2,4,5]$. The combustor was mounted on a three-axis translation stage and could be positioned with regard to the diagnostics setup. The coordinates used in the following discussions are defined in Fig.1. For this study, the combustor was operated with a total air flow rate of 315 SLM (standard liter per minute) at 1 bar and a preheating temperature of $300{ }^{\circ} \mathrm{C}$. Non-preheated methane was added 
into the main air through a static mixer to achieve an air equivalence ratio of $\lambda=1.4$. The thermal loading of the system was estimated to be $14 \mathrm{~kW}$. The average jet exit velocity was maintained at $150 \mathrm{~m} / \mathrm{s}$.

\subsection{Global measurements}

A commercial particle image velocimetry (PIV) system (LaVision Flowmaster, with $5 \mathrm{~Hz}$ repetition rate) was used to obtain a global view of the velocity fields in different sections of the combustor chamber. A small portion of the main air (non-preheated) was seeded with titanium dioxide $\left(\mathrm{TiO}_{2}\right)$ particles $(\mathrm{d}=1 \mu \mathrm{m})$ and injected into the static mixer. The second harmonic output of a dual-cavity Nd:YAG laser (with pulse energy of $2 \times 120 \mathrm{~mJ}$ ) was used to illuminate the particles. The laser beam was expanded into a sheet of $200-\mathrm{mm}$ tall and $1-\mathrm{mm}$ thick and was aligned through the center of the jet nozzle ( $\mathrm{x}-\mathrm{y}$ or $\mathrm{y}-\mathrm{z}$ plane, see Fig.1)). Mie scattering signal from the particles was collected into a CCD camera $(1376 \times 1040$ pixels $)$ coupled with a $\mathrm{f}=50 \mathrm{~mm}$ lens $(\mathrm{f} / 4)$, a narrow bandpass filter $(532 \pm 5 \mathrm{~nm})$ and a mechanical shutter (to suppress flame luminosity). Velocity vectors were calculated using a multipass adaptive window cross correlation algorithm with a final window size of $16 \times 16$ pixels (corresponding to a $2.2 \times 2.2 \mathrm{~mm}^{2}$ spatial resolution). Based on the \pm 0.1 pixel uncertainty of the cross correlation peak-finding algorithm, the uncertainty of the velocity measurements was estimated to be $\pm 2.3 \mathrm{~m} / \mathrm{s}$. Separately, $\mathrm{OH}^{*}$ chemiluminescence from the flame was imaged using a combination of a CCD camera, an intensifier, a UV lens (Cerco, $\mathrm{f}=45 \mathrm{~mm}, \mathrm{f} / 1.8$ ) and a bandpass filter centered at $310 \mathrm{~nm}$.

\subsection{Localized high-speed diagnostics}

To time-resolve the jet oscillation mentioned in Section 1 and its impact on combustion, a highspeed diagnostic system was employed. Velocity fields were measured using a stereoscopic PIV system at a repetition rate of $5 \mathrm{kHz}$. The stereo-PIV system was based on a dual-cavity Nd:YAG laser (Edgewave IS-6IIDE, $2.6 \mathrm{~mJ} /$ pulse at $532 \mathrm{~nm}$ ) and a pair of CMOS cameras (LaVision HSS8) with $\mathrm{f}=200 \mathrm{~mm}$ lenses (f/5.6). A $10 \mu$ s delay was set between the two PIV laser pulses, resulting in an estimated uncertainty of $\pm 0.2 \mathrm{~m} / \mathrm{s}$. A final field of view (FOV) of approximately $27 \times 25 \mathrm{~mm}^{2}$ was chosen to achieve a spatial resolution of $0.5 \times 0.5 \mathrm{~mm}^{2}$, with $16 \times 16$ pixels interrogation window.

Simultaneously with PIV, either $\mathrm{OH}^{*}$ chemiluminescence imaging or planar $\mathrm{OH}$ Laser-Induced Fluorescence (PLIF) was performed. For $\mathrm{OH}^{*}$ imaging, signal was collected via a UV lens (Cerco, $\mathrm{f}=45 \mathrm{~mm}, \mathrm{f} / 1.8$ ) coupled with a $310-\mathrm{nm}$ bandpass filter into an intensified CMOS camera (LaVision HSS6 with HS-IRO). For OH PLIF, a frequency-doubled dye laser (Sirah Credo) was pumped by the $532 \mathrm{~nm}$ output of an $\mathrm{Nd}$ :YAG laser (Edgewave IS-8IIE) to produce $\sim 100 \mu \mathrm{J} /$ pulse at $\sim 282 \mathrm{~nm}$. The laser was tuned to the peak of $\mathrm{Q}_{1}(7)$ line in the $\mathrm{OH} A-\mathrm{X}(1,0)$ band, which is insensitive to temperature in the range of $\mathrm{T}=1200-2000 \mathrm{~K}$ (with only $10 \%$ change in lower state population). The $\mathrm{OH}$ PLIF excitation pulse was placed temporally between the two 
Fig. 2

(a)\&(b) Average flow fields represented by streamlines color-coded by velocity magnitude in non-reacting and reacting flows, respectively

(c) Average $\mathrm{OH}$ chemiluminescence from the reacting flow at the operating condition

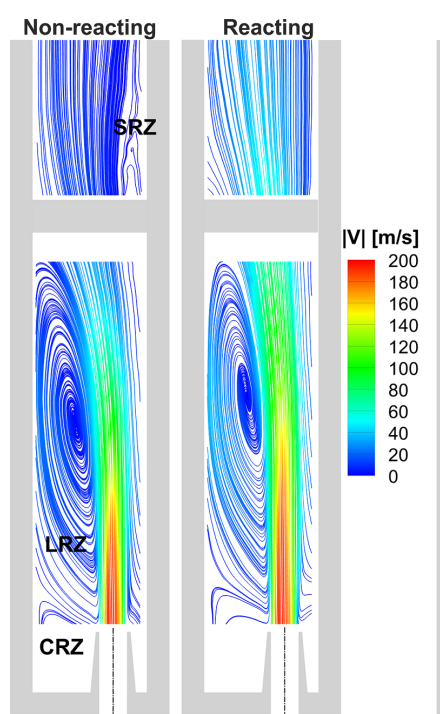

(b)

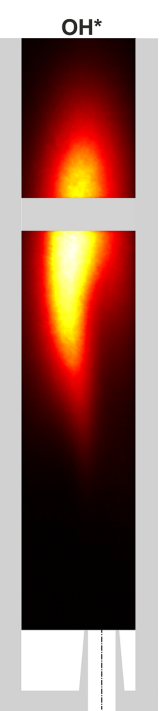

(c)

PIV pulses. Fluorescence signal was collected with the same camera system used for $\mathrm{OH}$ chemilunimescence imaging, with an intensifier gate width of $100 \mathrm{~ns}$ and an FOV of about $40 \times 40 \mathrm{~mm}^{2}$ to overlap with that of the PIV. The obtained OH PLIF images went through a post processing routine including (1) background (dark current) subtraction, (2) laser profile correction (by an averaged Acetone PLIF signal prior to each measurement) and (3) a 2 by 2 software binning to improve signal to noise. For both PIV and PLIF, the laser sheets were aligned through the center of the nozzle (x-y plane, see Fig.1), about $100 \mathrm{~mm}$ above the jet exit.

\section{General observations}

Figure 2 shows the average flow field and $\mathrm{OH}^{*}$ signal distribution measured at the operating condition from the front view (refer to Fig.1) with the diagnostic systems described in Section 2.2. Since the flame occupied mainly the bottom section and part of the middle section, the results have been cropped accordingly. Although the flow field of the bottom and middle sections were obtained from two separate measurements, a good consistency can be found between them. As reported in our previous work $[3,7]$, the dominant feature of this combustor is the lateral recirculation zone (LRZ) between the main jet and the chamber wall. This is evident from both the nonreacting (Fig.2(a)) and reacting (Fig.2(b)) cases. Two other recirculation zones can also be identified in Fig.2(a): a corner recirculation zone (CRZ) under the LRZ and a secondary recirculation zone (SRZ) on the opposite side of the jet. The average flow fields exhibit similar structures in both non-reacting and reacting cases, except for an overall higher velocity and the disappearance of the SRZ in the reacting case (Fig.2(b)). From Fig.2(c), it is clear that flame is lifted and stabilized at about midway of the bottom section, coinciding with the core of the LRZ. The flame also appears strongly asymmetric with respect to the nozzle axis, with a bias towards the LRZ. 
Fig. 3

Instantaneous flow fields from the bottom section of the combustor in the nonreacting case shown in Fig.2(a)

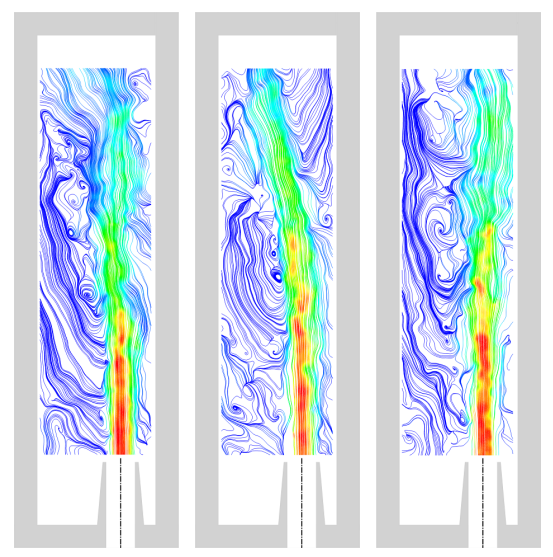

Figure 3 shows selected single-shot velocity fields from the lowspeed measurements (uncorrelated in time) in the bottom section of the combustor in the nonreacting case given in Fig.2(a). Rather than maintaining a constant position, the jet exhibits a varying extent of tilt towards either side of the combustor walls, which appears to exert an influence on the recirulation zones, notably the disappearance of the SRZ. The same phenomenon can be found in the reacting case as well. This kind of jet motion has been observed in previous measurements in FLOX ${ }^{\circledR}$ combustors. They have however been deemed as random turbulent events until the implementation of a highspeed PIV measurement in Ref.[7] in this single-nozzle combustor, which revealed its true nature as a periodic jet oscillation.

However, it is not immediately clear from the measurement results (1) what pattern the jet oscillation follows with this peculiar asymmetric jet setting, and (2) how exactly it affects the combustion process. The following sections are devoted to address these two questions by analyzing the measurement results following a systematic approach based on the proper orthogonal decomposition (POD) of the flow field.

\section{Self-sustained jet oscillation}

First, the global behavior of the jet oscillation is derived from the velocity measurements using the system described in Section 2.2. Second, the coupling between various jet oscillation modes is deduced from the high-speed PIV measurements described in Section 2.3. In the end, the differences between jet oscillation in nonreacting and reacting flows are discussed.

\subsection{Proper orthogonal decomposition (POD)}

POD is a well-established analytic tool for identifying coherent structures in unsteady flow fields [23]. POD establishes an orthonormal basis set consisting of POD Eigenmodes $\boldsymbol{\Phi}_{j}$, to optimally represent the fluctuating portion of the velocity field $\boldsymbol{u}^{\prime}$, so 
Fig. 4 Energy fraction of POD spatial modes of the non-reacting flow field

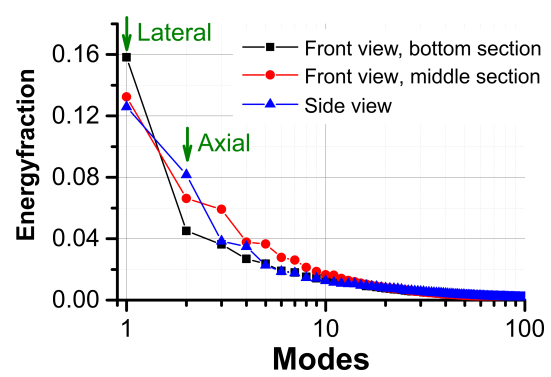

Fig. 5

POD spatial modes of the non-reacting flow field:

(a) Lateral mode of the front view

(b) Axial mode of the front view

(c) Lateral mode of the side view

(d) Axial mode of the side view

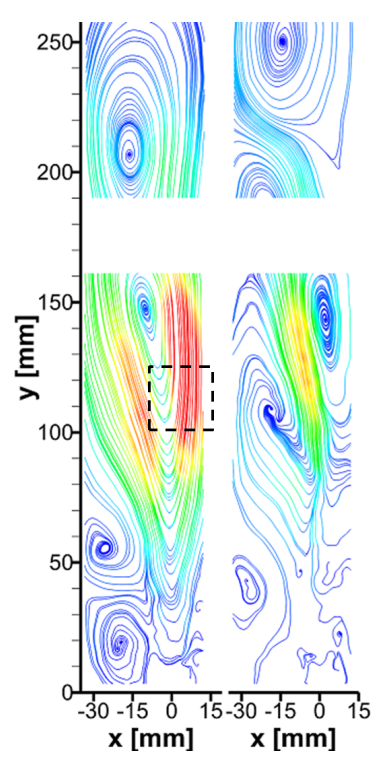

(a)

(b)

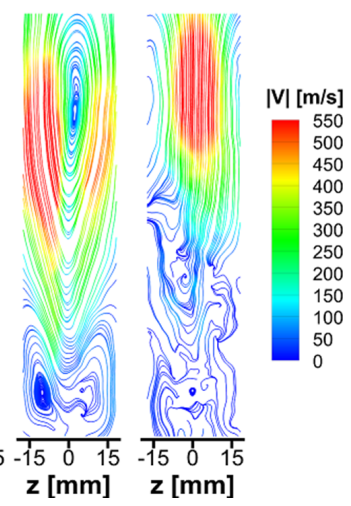

(d)

that the instantaneous flow field $\boldsymbol{u}_{i}$ can be reconstructed as:

$$
\boldsymbol{u}_{i}=\overline{\boldsymbol{u}}+\sum_{j=1}^{N} a_{i j} \cdot \boldsymbol{\Phi}_{j}
$$

where $\overline{\boldsymbol{u}}$ is the average velocity field, $a_{i j}=\left\langle\boldsymbol{u}_{i}^{\prime} \cdot \boldsymbol{\Phi}_{j}\right\rangle$ is the temporal coefficient of mode $j$. The contribution of mode $j$ to the total kinetic energy can be represented by its corresponding Eigenvalue $\lambda_{j}$ :

$$
\lambda_{j}=\frac{1}{N} \cdot \sum_{i=1}^{N} a_{i j}^{2},
$$

In this work, a snap-shot method of POD [24] was employed to derive $a_{i j}$ and $\boldsymbol{\Phi}_{j}$ by solving the Eigenvalue problem of the correlation matrix $K_{i j}=\left\langle\boldsymbol{u}_{i}^{\prime} \cdot \boldsymbol{u}_{j}^{\prime}\right\rangle$, constructed from $N$ snap shots [25]. The decomposition results of the global velocity fields are shown in Fig. 4 and 5.

Figure 5 shows the first two spatial Eigenmodes of the non-reacting flow field for both the front $(\mathrm{a} \& \mathrm{~b})$ and side views (c\&d) of the combustor. They were obtained from 
decomposing sets of 1000 global flow field measurements. For the measurements of the side view, the combustor was rotated by $90^{\circ}$, such that the laser sheet crossed the $y-z$ plane (see Fig.1). The contributions of the spatial modes to the total energy in the flow field are plotted in Fig.4. It is clear that the first mode is by a large margin the most dominant (referred to in this work as the 'lateral mode'). The lateral mode features a distribution of velocity peaks on each side of the jet. A similar spatial mode was obtained from a confined planar jet in Ref.[18] and was linked to periodic jet flapping. In contrast, the second mode ('axial mode') contains just a single velocity peak along the jet trajectory. The third and fourth modes (not shown in Fig.5) are the mode pairs to the lateral and axial modes. They have similar spatial structures as their stronger correspondents, but contribute much less to the total energy.

\subsection{Low-order modeling of flow field fluctuations}

Without prior knowledge, it is difficult to comprehend the POD spatial modes shown in Fig.5 and their connections to flow field fluctuations. Here, they are interpreted via a phase $(\psi)$-dependent low-order modeling detailed in Ref.[25]. It is carried out by utilizing the average flow field $(\overline{\boldsymbol{u}})$, the spatial Eigenmode $\left(\boldsymbol{\Phi}_{j}\right)$ of interest (e.g., lateral and axial modes) and assuming a sinusoidal oscillation:

$$
\boldsymbol{u}_{j}(\psi)=\overline{\boldsymbol{u}}+\sqrt{\lambda_{j}} \cdot \sin \psi \cdot \boldsymbol{\Phi}_{j}
$$

Note that Eq.3 is not used here to construct a first-order approximation of the flow field, but only to visualize flow oscillations described by each targeted spatial mode.

Figure 6 shows the phase-dependent non-reacting flow fields, modeled based on the lateral modes (Fig.5(a\&c)) for both the front and side views. The jet motion implied in the random single shots of the velocity field in Fig. 3 is fully represented here. In the front view (first four columns),from $\psi=0^{\circ}$ to $90^{\circ}$, the jet flaps leftwards while pushing the LRZ upstream. In the meantime, the SRZ, which is barely visible in the average flow field, increases in size and reaches its maximum at $\psi=90^{\circ}$. The rightward flapping continues into the second half cycle $\left(\psi=180^{\circ}\right.$ to $\left.360^{\circ} / 0^{\circ}\right)$, until the jet almost straightens up at $\psi=270^{\circ}$. This is also accompanied by the upward motion of the LRZ and the disappearance of the SRZ. From the side view (last four columns), the jet can also be seen flapping left and right, resulting in upward/downward motion of the recirculation zones on each side of the jet. This resembles more the flapping motion observed in symmetric planar jets [15].

The modeled flow fields based on the axial modes are shown in Fig.7. The middle section exhibited little variance over different phases and was therefore not included in the plot. From the velocity magnitude, it seems the axial mode is associated with a fluctuation causing the jet velocity in the far field to go through an increase/decrease cycle. However, the jet does not seem to be affected in the near field, suggesting that this axial fluctuation is not a pulsating motion along the nozzle axis. Rather, this axial fluctuation is most likely a manifestation of jet moving in and out of the observation plane, i.e., jet flapping motion perpendicular to the laser cut-through plane. If indeed the in-plane (lateral mode) and out-of-plane (axial mode) motion are of the same nature (i.e., all related to jet flapping), a coupling between the two can be expected. 


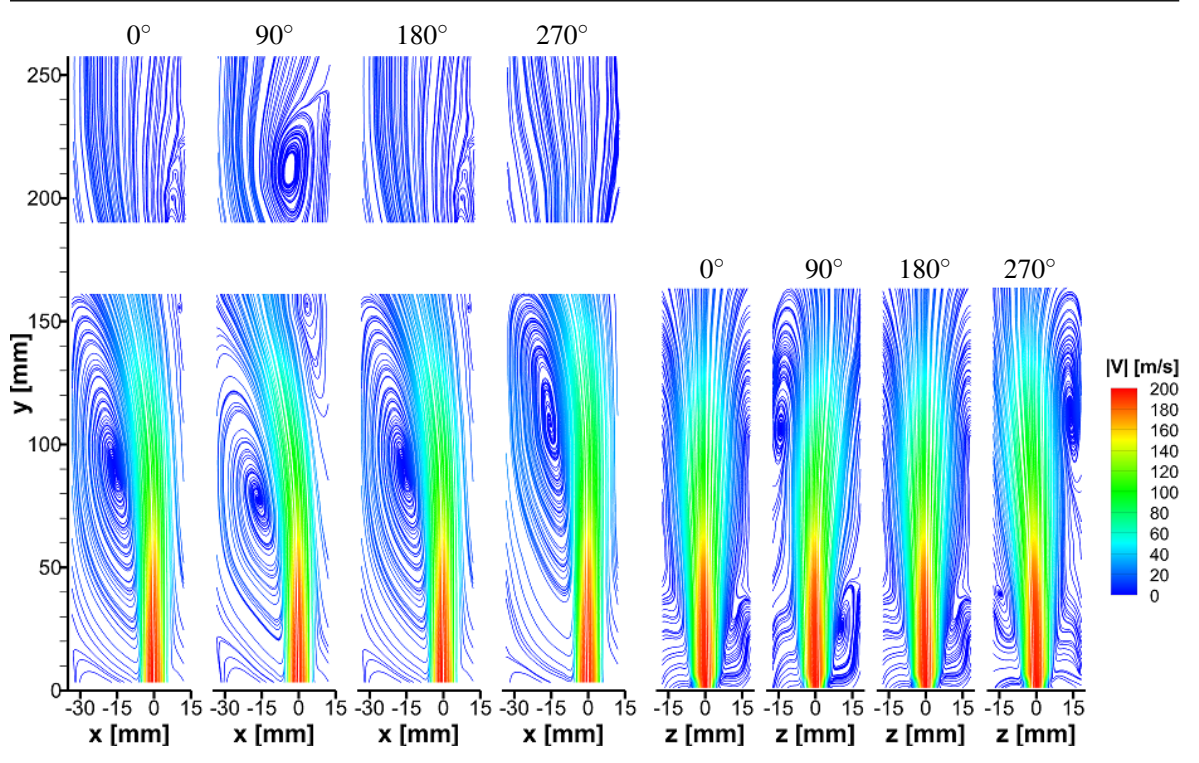

Fig. 6 Low-order modeling of phase-dependent flow fields based on the lateral modes of the non-reacting flow field for both the front view (first four columns) and the side view (last four columns)
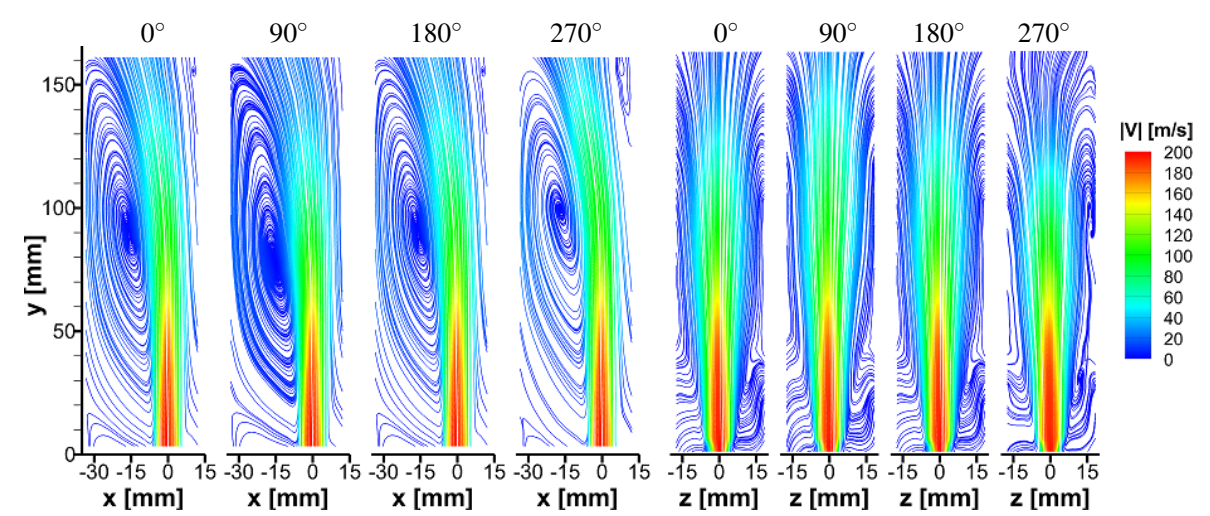

Fig. 7 Low-order modeling of phase-dependent flow fields based on the axial modes of the non-reacting flow field for both the front view (first four columns) and the side view (last four columns)

\subsection{Mode coupling}

As shown in [26], two spatial modes (representing the real and complex parts) are needed to describe a traveling wave. The flapping motion in a confined planar jet (a 2-D flow) was also shown to be controlled by two spatial modes with similar energy contributions but a phase shift of their temporal coefficients in the frequency domain [18]. As already mentioned in Section 4.1, the third and fourth spatial modes were found to have similar structures as the lateral and axial modes. To shed light on the 
Fig. 8

POD analysis of velocity fields measured at $5 \mathrm{kHz}$ in the non-reacting flow field: (a) Lateral mode and its companion (Lateral') of the front view and (b)\&(c) Frequency analysis of their temporal coefficients (a)
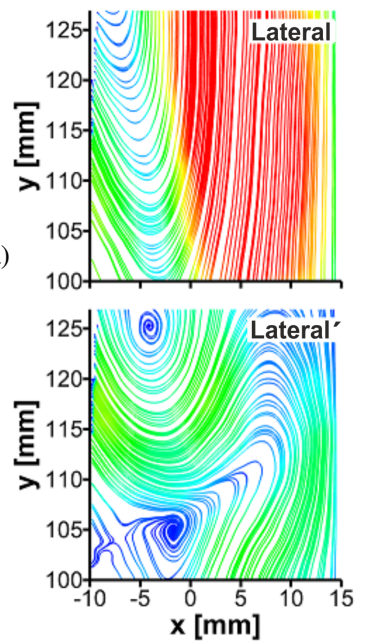

(b)

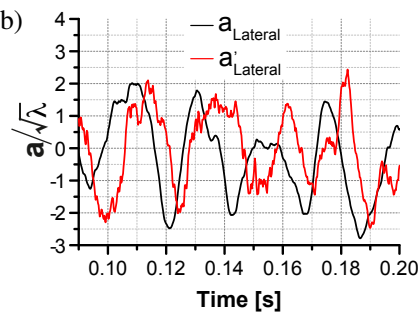

(c)

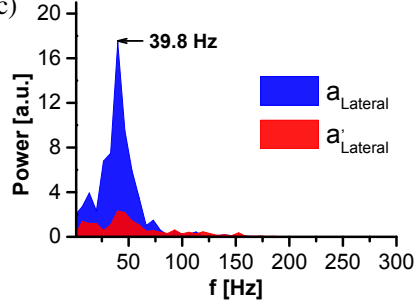

complex asymmetric and three dimensional jet motion in the combustor (evident from Fig.6 and 7), the temporal behaviors of various spatial modes and their interactions need to be examined. This is done by time-resolving the flapping motion using the $\mathrm{kHz}$ diagnostic system.

Figure 8(a) shows the lateral modes obtained from decomposing a set of 3750 shots of the velocity fields obtained at $5 \mathrm{kHz}$, plotted on the same color scale as Fig.5. The relative location of the FOV is indicated with a dashed box in Fig.5. Even though the FOV is much smaller, the spatial Eigenmodes and their energy contributions (omitted here) are consistent with the global flow field shown in Fig.4 and 5. The companion lateral mode, denoted by a prime sign, exhibits a more convoluted structure with much lower velocity magnitude. The correlation between the two modes is much clearer from their temporal coefficients ( $a$ in Eq.2). As shown in Fig.8(b), the weaker mode $\left(a_{\text {Lateral }}^{\prime}\right)$ lags behind the primary mode $\left(a_{\text {Lateral }}\right)$ by roughly $1 / 4$ of a cycle, as expected between a mode pair. From Fig.8(c), their similarity also extends into the frequency domain: the strongest peak in the power spectral density (PSD) of the temporal coefficient can be found at the same frequency. The same observations were made regarding the two axial modes, the results are therefore omitted here.

In addition to the coupling between the mode pairs, coupling between the lateral and the axial modes was also identified. From their temporal coefficients plotted in Fig.9(a) ( $a_{\text {axial }}$ was smoothed with a $\Delta t=0.01 \mathrm{~s}$ window to remove high frequency noise), the axial mode appears to have doubled the time period as the lateral mode. This is confirmed from PSD of their temporal coefficients given in Fig.9(b). The axial mode peaks at $19.9 \mathrm{~Hz}$, exactly half of the strongest peak found in the lateral mode. Furthermore, Fig.9(a) shows that the peaks and valleys in the axial mode (correspond to phase angle of $90^{\circ}$ and $270^{\circ}$ ) seem to always coincide with the peaks in the lateral mode. A Lissajous plot of their temporal coefficients in Fig.9(c) shows that the temporal coefficients do not scatter randomly in the phase portrait but rather appear to be bounded within a parabolic-like trajectory. 
Fig. 9

Coupling of the POD temporal coefficients between the lateral and axial modes of the front view in the non-reacting flow:

(a)\&(b) Comparison of their phase and frequency

(c) Lissajous plot of normalized coefficients

(d) Illustrative jet flapping motion (a)

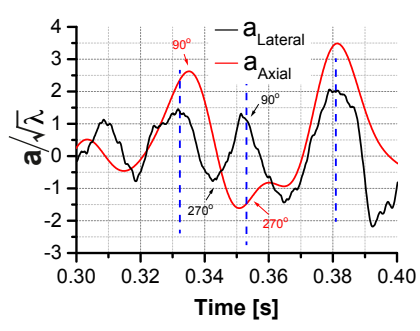

(b)

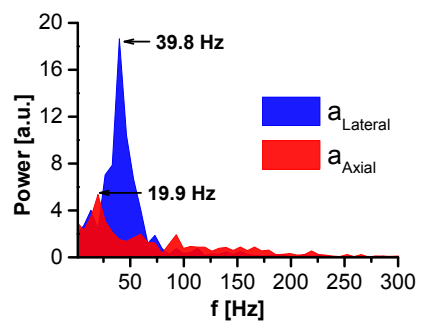

(c)

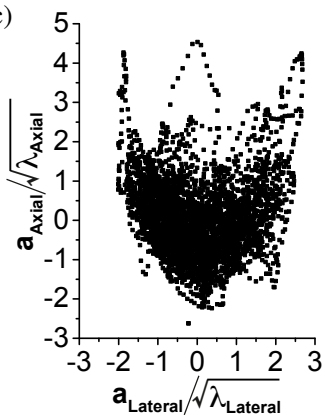

(d)

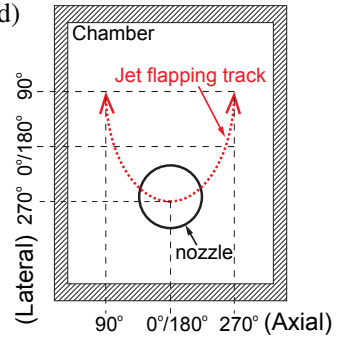

These results can be combined with the phase-modeling in Fig.6 and 7 to help reconstruct the 3-D jet flapping motion inside the combustor, as illustrated schematically in Fig.9(d). A likely projection of the jet flapping motion from the top view is represented by the red dashed line with regard to the jet nozzle. Based on Fig.9(a), the corresponding phase angles at various jet locations are also marked out with respect to the front and the side views of the combustor chamber (refer to Fig.1(d)). Since the axial mode is associated with in-and-out-of-plane motion of the jet, it can be viewed essentially as jet flapping in the side view. As the jet moves along the red dashed line, it completes two cycles of jet flapping from the perspective of the front view. While from the side view, it only flaps one cycle. This is consistent with the observations in Fig.9(a) and (b).

It should be emphasized that the simple jet flapping track illustrated in Fig.9(d) is only an idealized case. It is presented to demonstrate the 3-D nature of the jet oscillation inside the combustor chamber. As is obvious from the pseudo-sinusoidal behavior of the temporal coefficients in Fig.9(a) and the rather noisy phase portrait in Fig.9(c), jet flapping in different cycles can follow various tracks, which are not necessarily symmetric to the nozzle axis within the same cycle.

\subsection{Influence of reaction on the flow field instability}

As demonstrated in Section 3, small differences can be observed in the average flow field between non-reacting and reacting cases (see Fig.2). Furthermore, POD spatial modes exhibit nearly identical spatial distributions in both reacting and non-reacting cases, with the same ordering of energy contributions (thus omitted here). Similar 
Fig. 10

Coupling of the temporal coefficients between the lateral and axial modes of the front view in the reacting flow:

(a) Phase diagram

(b) Power spectra of the temporal coefficients

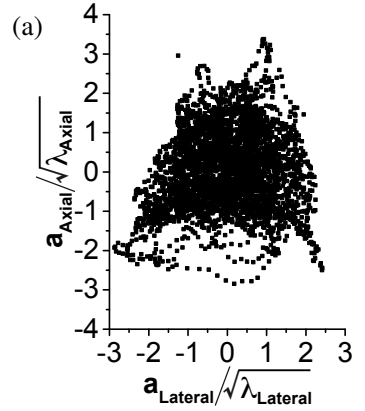

(b)

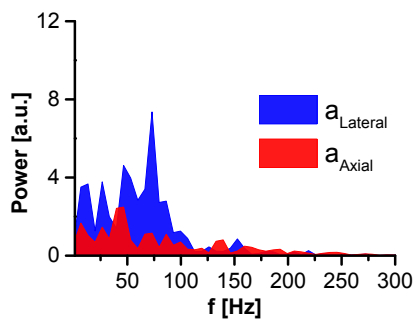

mode coupling between the lateral and axial modes also exists in the reacting case, but appears mirrored (i.e., with a $180^{\circ}$ phase shift) from the non-reacting case, as shown in Fig.10. However, the temporal coefficients appear to have multiple peaks in the frequency domain, as plotted in Fig.10(b). Their amplitudes are also much lower than in the non-reacting case.

This kind of coexistence of various frequencies in jet oscillation has been previously documented [13]. It is clear that in the reacting flow, jet oscillation becomes less coherent. After a close look at the time-resolved experimental results, these different peaks can all be related to the same general motion of jet flapping (as modeled in Fig.6), but at different pace (i.e., oscillation period). The multi-frequency feature observed here could stem from a feedback between the jet oscillation and combustion heat release. Jet oscillation may alter flame structure and flame lift-off height, which may then trigger a change in local heat release, leading to a shift of the density balance in the combustor chamber and subsequently affecting the jet oscillation.

\section{Jet oscillation induced combustion instabilities}

The effect of jet oscillation on the combustion process postulated above based on the the frequency analysis in Fig.10(b) is scrutinized in this section. With the help of the temporally correlated data from both velocity and scalar fields $(\mathrm{OH}$ chemiluminescence and OH PLIF signal) measured at $5 \mathrm{kHz}$, we can reconstruct the fluctuations without relying on the low-order modeling presented in Section 4.2. The results were first sorted based on phase angles extracted from the temporal coefficients of the lateral mode, such as the ones shown in Fig.8(b). A zero-crossing detection algorithm was used to assign $0^{\circ}$ or $180^{\circ}$ to corresponding frames, assuming a sinusoidal behavior of the temporal coefficients (to match the phase angles used in Section 4.2). Then the phase angles were extrapolated accordingly for frames between zero crossings. A low pass filter was introduced in the zero-crossing algorithm to discard frames that fall into fluctuations larger than $100 \mathrm{~Hz}$ (treated as noise). An entire cycle was then divided into eight groups, each spanning a range of $45^{\circ}$. This information was then used for phase averaging.

Note that the influence of the axial mode was neglected, due to its much less contribution to the total fluctuation (from the energy fractions of the POD modes, see Fig.4). 


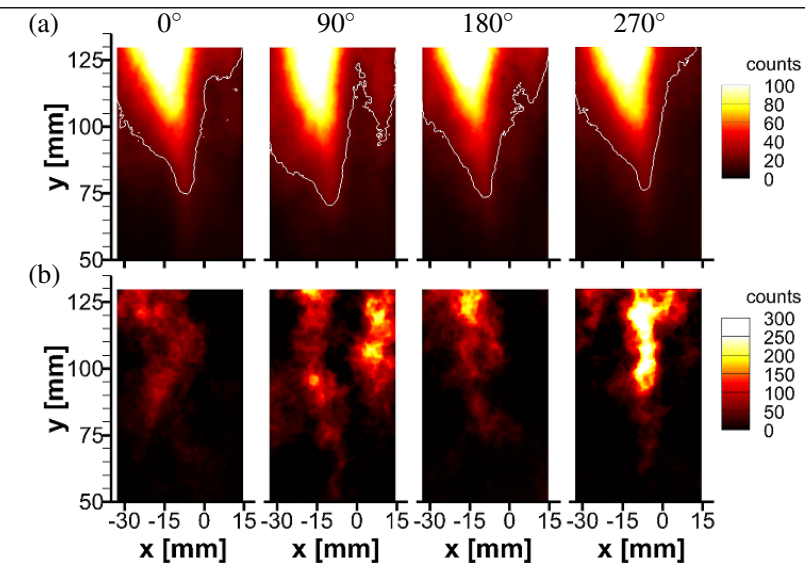

Fig. $11 \mathrm{OH}$ chemiluminescence images from the 5-kHz measurements at different phase angles: (a) phase averaged based on the temporal coefficients of the lateral mode (b) representative instantaneous shots from different phase groups

\subsection{Influence on flame shape and flame lift-off height}

From simultaneous measurements of PIV and $\mathrm{OH}$ chemiluminescence imaging at $5 \mathrm{kHz}, \mathrm{OH}^{*}$ images were phase averaged using the method described above. Figure 11(a) shows phase-averaged $\mathrm{OH}^{*}$ signal distributions at the four representative phase angles. Only part of the bottom section of the combustor was imaged to focus on the root of the flame. The contour line at signal counts of 20 was also drawn to better showcase the variation in flame shape. As the jet flaps left to reach phase $90^{\circ}$, the flame lift-off height reduces slightly and flame becomes more attached to the left wall. Also, it extends along the right wall upstream, corresponding to the increase of SRZ shown in Fig.6. On the contrary, during the second half of the cycle, as the jet straightens up, flame becomes more detached from the left wall and its lift-off height also increases slightly. Flame near the right wall recedes downstream, coinciding with the disappearance of the SRZ in the second half cycle of jet flapping shown in Fig.6.

In fact, the flame root is more dynamic than the phase averaged images may suggest. This is demonstrated in Fig.11(b), in which representative instantaneous $\mathrm{OH}$ chemiluminescence images from each phase group are shown. The flame root varies notably from their corresponding phase average. As discussed in Section 4.4, jet flapping in the reacting case can change from cycle to cycle or even in the same cycle. Its influence on the flame structure can therefore vary even in the same phase group. Therefore, phase averaging could have subdued the extreme variations in certain cases.

In order to gain insight into the mechanisms of flame variations shown in Fig.11, flow-flame interactions were examined at a finer scale with the results from simultaneous PIV and OH PLIF measurements at $5 \mathrm{kHz}$. Figure 12(a) overlaps the phase averaged velocity field (streamlines) with $\mathrm{OH}$ signal distribution. The plot at each phase is stitched together from two separate measurements, although good conver- 
Fig. 12

Results from 5-kHz measurements, phase averaged based on the temporal coefficients of the lateral mode:

(a) Velocity field and OH PLIF signal distribution

(b) Contours of velocity magnitude

$0^{\circ}$

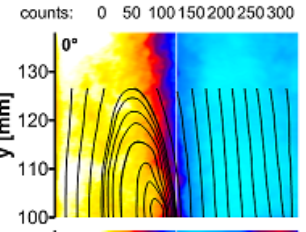

$[\mathrm{V}][\mathrm{m} / \mathrm{s}]: \quad 0 \quad 20406080100$

$90^{\circ}$

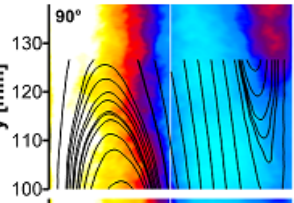

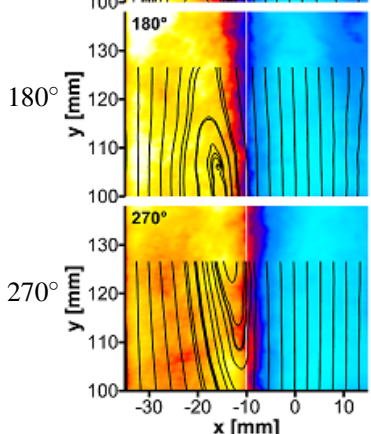

(a)

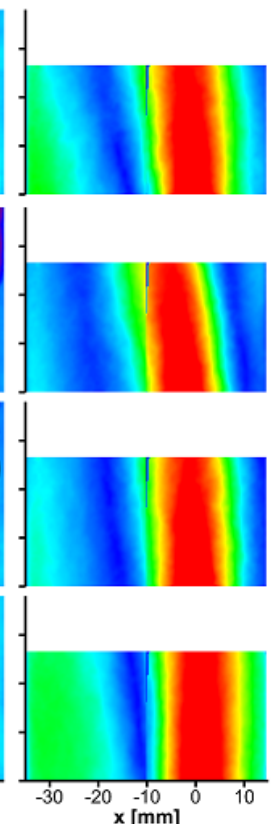

(b)

gence can be observed at the boundaries of the FOVs (at $x=-10 \mathrm{~mm}$ ). From top to bottom of Fig.12(a), the jet is seen to swing left and right, accompanied by the movement of the LRZ and appearance/disappearance of SRZ, corresponding well with the low-order modeling of the flow field shown in Fig.6. OH PLIF signal distribution also responses to jet flapping. Compared with phases $0^{\circ}$ and $180^{\circ}, \mathrm{OH}$ signal concentrates more towards the left wall at $90^{\circ}$, while appearing in general weaker and more distributed at phase $270^{\circ}$. This agrees with the trend seen in $\mathrm{OH}$ chemiluminescence in Fig.11, where flame appears at this location more attached to the left wall at phase $90^{\circ}$ but becomes detached and more lifted at $270^{\circ}$. Near the right wall, $\mathrm{OH}$ intensity increases considerably as the SRZ appears, also consistent with the trend seen in $\mathrm{OH}$ chemiluminescence in Fig.11.

Figure 12(b) plots the magnitude contour of the velocity fields shown in Fig.12(a). It is clear that $\mathrm{OH}$ signal intensity increases with the size of the low-speed region found in the core of the LRZ. This is likely due to an enhanced mixing of the entrained and fresh gas mixtures in the low-speed regions, which act as anchoring points for the flame. As the LRZ moves upstream and downstream due to jet flapping (see Fig.6), so does its core and the flame stabilization point, leading to the shift of flame lift-off height. In a similar manner, the SRZ provides additional flame stabilization points near the right wall, allowing the flame to temporarily extend upstream at phase $90^{\circ}$.

The flow-flame interactions in the core of the recirculation zones (i.e., low-speed regions) are further inspected in the following. Instantaneous velocity fields and $\mathrm{OH}$ signal distributions, randomly selected from two extreme phase groups $\left(90^{\circ}\right.$ and $270^{\circ}$ ), are overlapped to illustrate the flow-flame interactions in the LRZ and SRZ. 


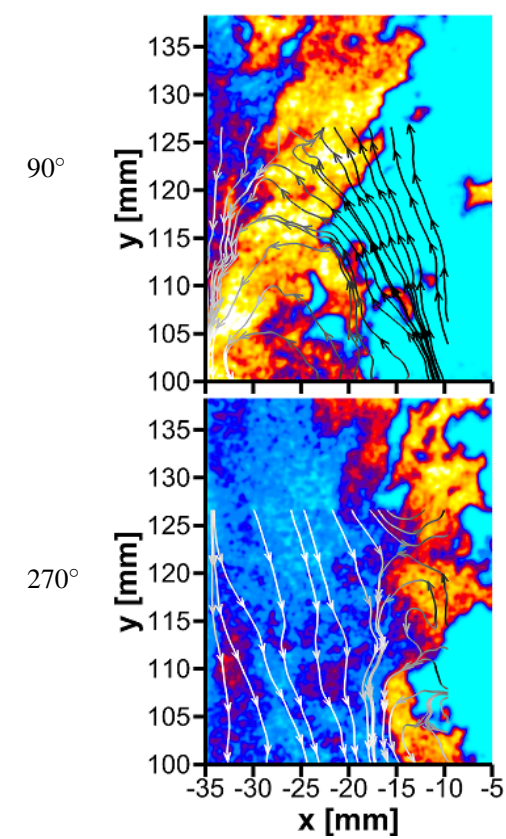

(a)

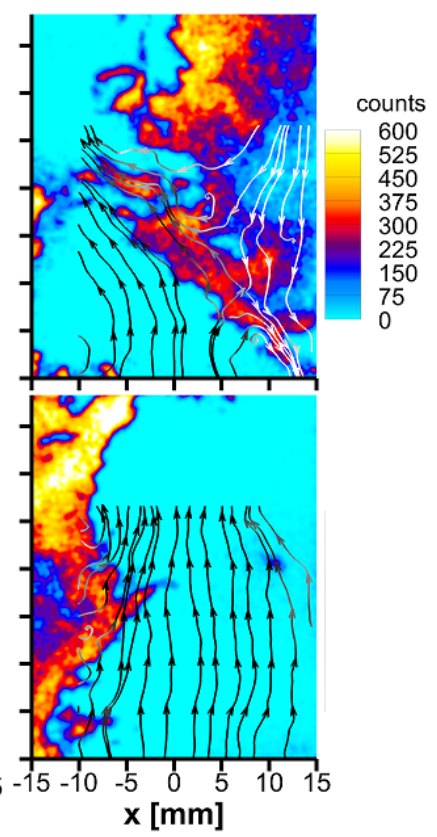

(b)

Fig. 13 Instantaneous velocity field and $\mathrm{OH}$ distribution chosen from phase groups of $90^{\circ}$ and $270^{\circ}$.

Note that Fig.13(a) and (b) are from two different sets of measurements. The regions with low $\mathrm{OH}$ signal are mostly accompanied by back flow, suggesting that they are mainly recirculated burned gas. On the other hand, regions with distinctively high $\mathrm{OH}$ signal (most likely from superequilibrium $\mathrm{OH}$ ) can be found between burned and unburned gas. Such sharp gradient in $\mathrm{OH}$ signal can be used as a qualitative marker of reaction zones. As can be seen, the reaction zones tend to distribute along the junctions of the high-velocity jet and the back flow, where opposing flow or vortices can be identified. These regions (gray streamlines) are characterized by low flow velocity, corresponding to the low-speed regions in the phase averaged fields in Fig.12(b). Similar observations can be made regarding the SRZ, as shown in Fig.13(b). As the SRZ forms when the jet flaps leftwards $\left(90^{\circ}\right)$, an additional channel for burned gas entrainment and low-speed mixing region is created opposite to the main flame stabilization region (i.e., LRZ). Such channel is completely closed when the jet flaps to the upright position at $270^{\circ}$.

\subsection{Influence on hot gas entrainment}

Not obvious from the above analysis is that jet flapping also controls the amount of hot gas entrainment into the LRZ, with more restricted back flow at phase $90^{\circ}$. This is demonstrated here through an extreme scenario of temporary blockage of the entrainment due to jet impingement, which can cause local extinction of the flame. 


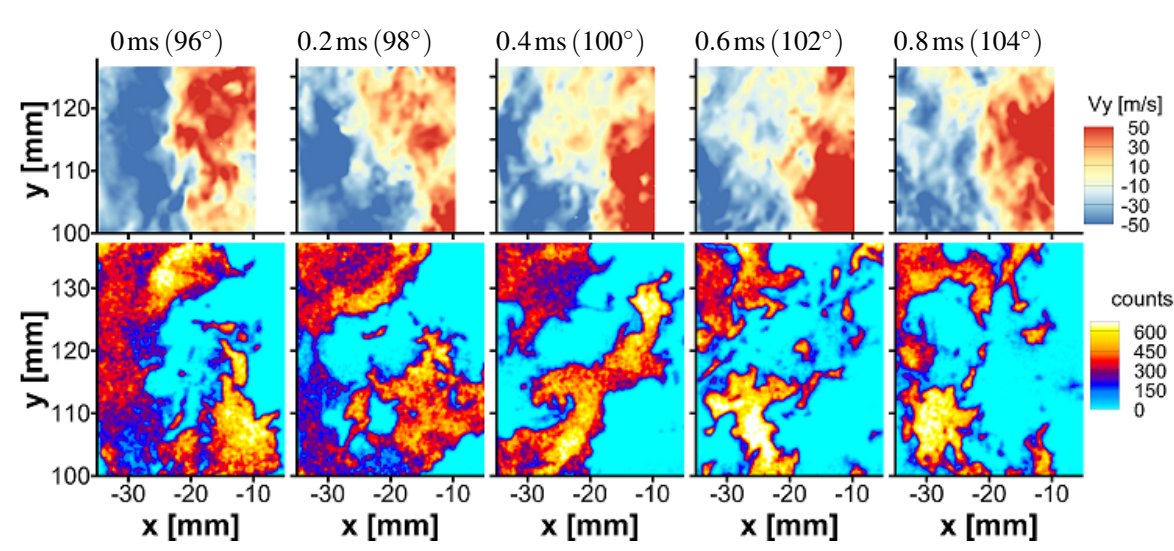

Fig. 14 Sequence of instantaneous velocity fields and $\mathrm{OH}$ distributions depicting a flame fragmentation process due to jet flapping

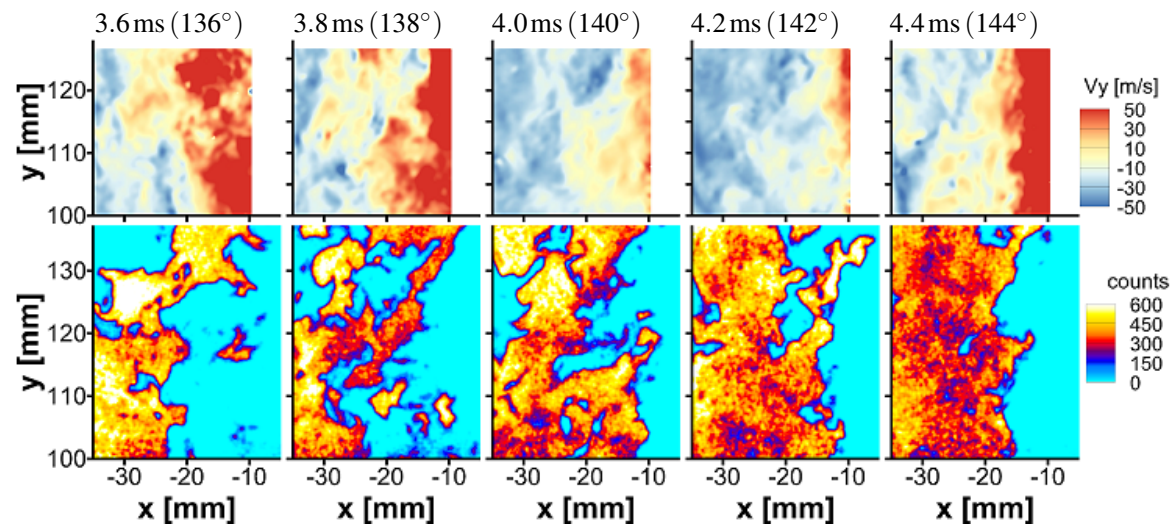

Fig. 15 Sequence of instantaneous velocity fields and $\mathrm{OH}$ distributions depicting a flame recovery process after temporary local extinction due to jet flapping (following Fig.14)

A sequence of instantaneous velocity fields and $\mathrm{OH}$ signal distributions is shown with an arbitrary time zero and their corresponding phase angles labeled above each frame (all from the group of phase $90^{\circ}$ ). The velocity fields are represented by the $y$ component to better illustrate the location of the jet and the back flow. As can be seen, when the jet flaps leftwards, it impinges on the wall and causes a partial blockage of the back flow. At the same time, the continuous region of $\mathrm{OH}$ signal (representing a combination of burned gas and reaction zones) first break into two parts following the impingement, and then disintegrates into fragments. Such fragmentation suggests that flame is no longer sustained in the FOV, but rather goes through local extinction.

However, the back flow is able to recover quickly in the FOV following the detachment of the jet, as depicted by a later sequence of frames in Fig.15. This sequence happens approximately $3 \mathrm{~ms}$ after those in Fig. 14 and belongs to the next phase group of $135^{\circ}$. From Fig.15, the reentry of burned gas into the LRZ results in significant 
increase of $\mathrm{OH}$ signal in the region. This could be attributed to rather abundant unburned gas retained in this region after the temporary local extinctions. Gradually, $\mathrm{OH}$ signal occupies the entire region left of the jet, signaling a restabilization of the flame in the FOV. Also notice that, the lack of reaction zones at $4.4 \mathrm{~ms}$ indicates that it is mainly burned gas in the FOV. Flame is most likely lifted off further downstream, consistent with the observation of phase sorted $\mathrm{OH}^{*}$ distribution in Fig. 11 from $90^{\circ}$ to $180^{\circ}$.

\section{Conclusions}

This paper presented experimental studies of a confined, premixed, preheated methaneair turbulent flame, generated in a single-nozzle FLOX ${ }^{\circledR}$ combustor. The results are summarized below.

First, based on proper orthogonal decomposition (POD) of measured flow fields using particle image velocimetry (PIV), periodic jet flapping is identified as the primary jet oscillation pattern inside the combustor chamber. It is found to modify the size and location of the lateral recirculation zone (LRZ) and the secondary recirculation zone (SRZ) located on each side of the jet.

Second, from spatially and temporally correlated measurements of velocity and $\mathrm{OH}$ chemiluminescence or planar laser-induced fluorescence of $\mathrm{OH}$ radicals (PLIF), jet flapping is demonstrated to impact combustion stability mainly via two mechanisms:

1) By influencing the LRZ and the SRZ as described above, jet flapping changes the size and location of the regions with opposing flow and vortices, which enhances mixing between the entrained hot gas and the unburned gas, consequently modifying the flame shape and flame lift-off height.

2) Jet flapping also controls the amount of hot gas entrainment into the LRZ. In extreme cases, jet impingement on the wall can cause temporary blockage of the back flow, leading to local flame extinction.

Since jets under confinement are susceptible to self-induced oscillations, other jet-stabilized combustors, especially those designed based on the FLOX ${ }^{\circledR}$ concept could also be affected in similar ways as demonstrated in this work. In combustors with multi-nozzle configurations, jet oscillation may facilitate flames sustained by the individual jets to interact with each other. This could further complicate the role of jet flapping on combustion stability.

Acknowledgements Zhiyao Yin acknowledges the financial support within the Helmholtz Postdoc Programme (Grant PD-112).

\section{References}

1. R. Lückerath, W. Meier, M. Aigner, J. Eng. Gas Turbines Power 130(1), 011505 (2008) 
2. O. Lammel, H. Schütz, G. Schmitz, R. Lückerath, M. Stöhr, B. Noll, M. Aigner, M. Hase, W. Krebs, J. Eng. Gas Turbines Power 132(12), 121503 (2010)

3. O. Lammel, M. Stöhr, P. Kutne, C. Dem, W. Meier, M. Aigner, J. Eng. Gas Turbines Power 134(4), 041506 (2012)

4. T. Roediger, O. Lammel, M. Aigner, C. Beck, W. Krebs, J. Eng. Gas Turbines Power 135(3), 031503 (2013)

5. O. Lammel, T. Rödiger, M. Stöhr, H. Ax, P. Kutne, M. Severin, P. Griebel, M. Aigner, in ASME Turbo Expo 2014: Turbine Technical Conference and Exposition (American Society of Mechanical Engineers, 2014), pp. V04BT04A031V04BT04A031

6. A. Zizin, O. Lammel, M. Severin, H. Ax, M. Aigner, in ASME Turbo Expo 2015: Turbine Technical Conference and Exposition (American Society of Mechanical Engineers, 2015), pp. V04AT04A050-V04AT04A050

7. Z. Yin, P. Nau, I. Boxx, W. Meier, in ASME Turbo Expo 2015: Turbine Technical Conference and Exposition (American Society of Mechanical Engineers, 2015), pp. V04BT04A017-V04BT04A017

8. H. Seliger, A. Huber, M. Aigner, in ASME Turbo Expo 2015: Turbine Technical Conference and Exposition (American Society of Mechanical Engineers, 2015), pp. V04BT04A004-V04BT04A004

9. M. Di Domenico, P. Gerlinger, B. Noll, in ASME 2011 Turbo Expo: Turbine Technical Conference and Exposition (American Society of Mechanical Engineers, 2011), pp. 519-530

10. F. Proch, A. Kempf, Proc. Combust. Inst. 35, 3337 (2015)

11. J. Wünning, J. Wünning, Prog. Energy Combust. Sci. 23(1), 81 (1997)

12. A. Cavaliere, M. de Joannon, Prog. Energy Combust. Sci. 30(4), 329 (2004)

13. A. Maurel, P. Ern, B. Zielinska, J. Wesfreid, Phys. Rev. E 54(4), 3643 (1996)

14. S.V. Gordeyev, F.O. Thomas, J. Fluid Mech. 414, 145 (2000)

15. N. Lawson, M. Davidson, J. Fluids Struct. 15(1), 59 (2001)

16. J. Mi, G. Nathan, R. Luxton, Flow Turbul. Combust 67(1), 1 (2001)

17. B. Kong, M. Olsen, R. Fox, J. Hill, Exp. Fluids 50(6), 1473 (2011)

18. O. Semeraro, G. Bellani, F. Lundell, Exp. Fluids 53(5), 1203 (2012)

19. G. Nathan, S. Hill, R. Luxton, J. Fluid Mech. 370, 347 (1998)

20. J. Mi, G. Nathan, J. Fluids Struct. 19(6), 851 (2004)

21. J. Mi, G. Nathan, C. Wong, J. Fluids Struct. 22(1), 129 (2006)

22. G. Nathan, J. Mi, Z. Alwahabi, G. Newbold, D. Nobes, Prog. Energy Combust. Sci. 32(5 - 6), 496 (2006)

23. G. Berkooz, P. Holmes, J.L. Lumley, Annu. Rev. Fluid Mech. 25(1), 539 (1993)

24. L. Sirovich, Q. Appl. Math. 45(3), 561 (1987)

25. M. Stöhr, R. Sadanandan, W. Meier, Exp. Fluids 51(4), 1153 (2011)

26. D. Rempfer, H.F. Fasel, J. Fluid Mech. 260, 351 (1994) 